\title{
Role of HPV-16 in Pathogenesis of Oral Epithelial Dysplasia and Oral Squamous Cell Carcinoma and Correlation of p16INK4A Expression in HPV-16 Positive Cases: An Immunohistochemical Study
}

\author{
Gaurav Pralhad Agrawal, ${ }^{1}$ Priya Shirish Joshi, ${ }^{2}$ and Anshita Agrawal ${ }^{3}$ \\ ${ }^{1}$ Department of Oral Pathology and Microbiology, SMBT Dental College and Hospital, Sangamner 422605, India \\ ${ }^{2}$ Department of Oral Pathology and Microbiology, Vasantdada Patil Dental College and Hospital, Sangli 416416, India \\ ${ }^{3}$ Department of Oral Pathology and Microbiology, Vananchal Dental College and Hospital, Garhwa, India \\ Correspondence should be addressed to Gaurav Pralhad Agrawal; agrawalgaurav28@rediffmail.com
}

Received 15 January 2013; Accepted 14 February 2013

Academic Editors: M. Mokni, A. Sonzogni, and A. Stringer

Copyright (C) 2013 Gaurav Pralhad Agrawal et al. This is an open access article distributed under the Creative Commons Attribution License, which permits unrestricted use, distribution, and reproduction in any medium, provided the original work is properly cited.

\begin{abstract}
The objective of current study is to evaluate the role of HPV-16 in the pathogenesis of oral epithelial dysplasias (OED) and oral squamous cell carcinoma (OSCC) by immunohistochemistry (IHC) and to know whether HPV-16 participates in disruption of the regulation of p16 INK4A suppressor protein in OED and OSCC by IHC. Histopathologically diagnosed 20 cases of OED and 20 cases of OSCC were selected from amongst the patients attending the OPD of Vasantdada Patil Dental College and Hospital, Sangli. Biopsy tissue section were then tested for HPV-16 by IHC. HPV-16 positive tissue sections were then again tested by p16 by IHC. Overall $22.5 \%$ of cases in our study were found to be positive for HPV 16 which includes $10 \%$ of cases of OED and 35\% cases of OSCC. Amongst the HPV 16 positive cases, more than $60 \%$ of cells were positive for p16INK4A IHC in OED (50\%) and OSCC (85.71\%). Thus, HPV 16 participates in disruption of the regulation of p16INK4A suppressor protein and can be used as surrogate biomarker for detection of HPV infection in OED and OSCC.
\end{abstract}

\section{Introduction}

Cancer is a disease of cells that escape the control mechanisms of orderly cell growth and acquire the ability to proliferate, invade normal tissues and metastasize [1]. Head and neck cancer is the sixth most common cancer worldwide, with an estimated annual burden of 563,826 incident cases (including 274,850 oral cavity cancers, 159,363 laryngeal, and 52,100 oropharyngeal cancers) [2,3]. In India oral cancer constitute around $9.8 \%$ of total cancer cases \& ranks first among the all cancer cases in males \& third most common among females. Age standardized incidence rate varies between 7 and 17/1000,000 person-years. The incidence rate is higher than the rate of 3 to $4 / 1000,000 /$ year found in western countries [4-6]. India has one of the world's highest incidences of head and neck cancers which were primarily caused by tobacco habits and alcohol consumption [5].
The association between high risk human papillomavirus [HR HPV] and risk of oral cancer development dates to back 1983, when HPV16 was detected in oral squamous cell tumors. Since then HPV DNA has repeatedly been found in a variable proportion of head and neck cancers [6-9].

HPV is a $\sim 7.9 \mathrm{~kb}$, nonenveloped, double-stranded, circular DNA virus that has been implicated in a variety of anogenital \& aerodigestive diseases, ranging from common warts to laryngeal papilloma to cervical cancer. The first isolation of this virus particle was performed in 1933 in rabbit papillomatosis [10].

The oral cavity is covered by an epithelium that has characteristics similar to those of the genital region, and both are exposed to strange bodies and microorganisms, some of which could be potentially carcinogenic. Considering that in the genital region this virus is implicated in the development of cancer of uterine cervix, it is possible that this link would 
also be present in relation to oral cancer. Therefore it has become important to know the frequency of this virus in the oral cavity and its probable relationship with the presence of the premalignant and malignant neoplasm in the region. Papillomavirus are considered to be persistent viruses capable of residing latently within host cells for extended periods of time [6].

The oncogenic potential of HPV is thought to be due to two oncoproteins, E6 and E7 [11-13]. The E6 protein of oncogenic HPV strains has been shown to interact with the p53 protein and promote its degradation by means of a ubiquitin-dependent pathway. The E7 oncoprotein can similarly complexed with retinoblastoma $(\mathrm{Rb})$ and inactivate it.

There are plethora of molecular techniques used for detection [14], which includes

(i) low sensitivity assays: immunoperoxidase, immunofluoresecence, and in situ hybridisation (ISH),

(ii) moderate sensitivity assays: Southern blot test, Dot blot test, and Hybrid capture 2 [15],

(iii) high sensitivity assays: polymerase chain reaction (PCR).

Any single method of detection has some limitations. Thus, use of algorithms that combine the strength of complementary assays is needed. The recent reports have suggested that overexpression of the p16INK4A protein can act as a surrogate biomarker of HPV-induced carcinomas [16]. HPVinduced carcinogenesis is associated with low $\mathrm{pRb}$ protein levels which leads to subsequent p16 upregulation. Positive p16INK4A immunostaining of HPV-associated tumors is $100 \%$ sensitive but only $79 \%$ specific $[3,17]$.

Considering all the previous facts this study focused on combining specificity of HPV immunohistochemistry with sensitivity of p16 immunohistochemistry for detection of role of HPV 16 in pathogenesis of OED \& OSCC.

\section{Aims and Objective}

(I) To evaluate the role of HPV-16 in the pathogenesis of oral epithelial dysplasias (OED) and oral squamous cell carcinoma (OSCC) by immunohistochemistry (IHC).

(II) To evaluate whether HPV-16 participates in disruption of the regulation of p16INK4A suppressor protein in OED and OSCC by IHC.

\section{Materials and Methods}

Histopathologically diagnosed 20 cases of OED and 20 cases of OSCC constituted the study group. These cases were clinically diagnosed either as oral precancer or cancer at Department of Oral Medicine and Radiology of our institute. Patients were referred for biopsy. The biopsy along with the patient's personal history and clinical details were collected from biopsy requisition form at Department of
Oral Pathology \& Microbiology and are summarized in the results. Histopathologic diagnosis of precancer or cancer was confirmed. The selected 40 cases were then screened immunohistochemically for HPV 16 positivity using primary antibody for HPV 16 from immunoglobulin fractions, diluted in PBS, pH 7.6, Clone: Cam Vir -1, Biogenix, CA \& Super Sensitive Polymer HRP Ready to Use kit. Positive control were selected (Figure 1).

$\mathrm{H} \& \mathrm{E}$ stained slides of selected cases were graded for epithelial dysplasia according to WHO 2005 classification of oral epithelial dysplasia [18] \& OSCC cases graded on the basis of differentiation as well, moderately \& poorly differentiated. They were further classified by TNM system as stages I, II, III, \& IV [19, 20].

3.1. Assessment: Immunohistochemically Stained Slides for HPV 16 \& $16 I N K 4 A$. Slides were interpreted on the basis of nuclear staining or combined nuclear \& cytoplasmic staining. Only cytoplasmic staining was considered as negative. Positivity was confirmed by comparison with positive control for HPV 16.

HPV 16 positive tissue sections were then tested for expression of p16INK4A by IHC. Primary antibody used was p16INK4A with immunoglobulin fraction diluted in PBS. Clone Used: G175-405, Mouse Monoclonal, Biogenix, CA.

The German semiquantitative scoring system $[16,19,20]$ was adopted with some modification for scoring the staining results of p16. All the slides were interpreted for nuclear as well as cytoplasmic positivity.

Every tumor was given a score according to the extent of stained cells as $0 \%=0,1-10 \%=1,11-50 \%=2,51-80 \%=3$, $81-100 \%=4$ :
(a) negative means $0 \%$ staining area,
(b) focally positive means $1 \%-80 \%$ area staining,
(c) diffusely positive means $81 \%-100 \%$ area staining.

\section{Results and Statistical Analysis}

Our study showed that in 20 cases of OED, 2 (10\%) cases were positive for HPV 16 whereas out of 20 cases of OSCC, 7 (35\%) cases were positive for HPV 16.

Total HPV 16 positivity including OED and OSCC was found to be $22.5 \%$.

All the observations and results were categorized as

(1) HPV 16 associations in OED,

(2) HPV 16 associations in OSCC,

(3) HPV 16 positivity and p16INK4A Expression.

4.1. HPV 16 Associations in Oral Epithelial Dysplasias. The study constituted 20 cases of OED. The age range was $20 \mathrm{yrs}$ to 75 yrs with mean age of 52.58 yrs. Out of total cases, $40 \%$ cases were in age group of 51 to 60 yrs. HPV 16 positivity was found to be mainly in 51 to 70 yrs of age group and this finding was statistically significant.

Genderwise distribution of cases of OED showed female predominance in HPV 16 positivity with 2 out of 3 females 
HPV-16

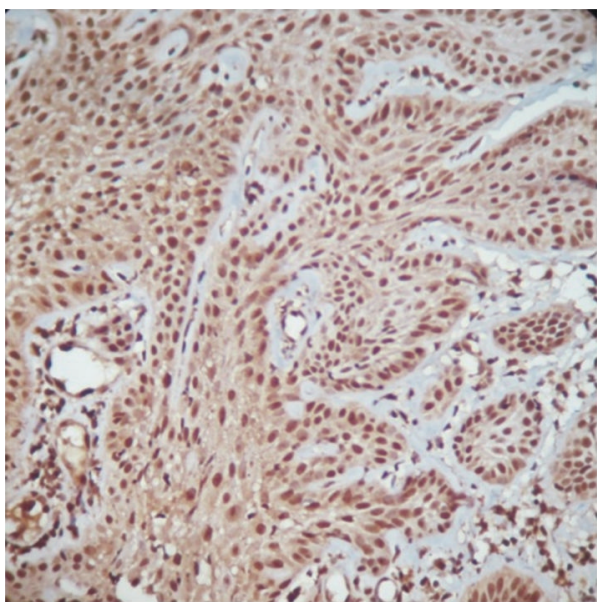

Positive control

$\mathrm{P} 16^{\mathrm{INK} 4 \mathrm{~A}}$

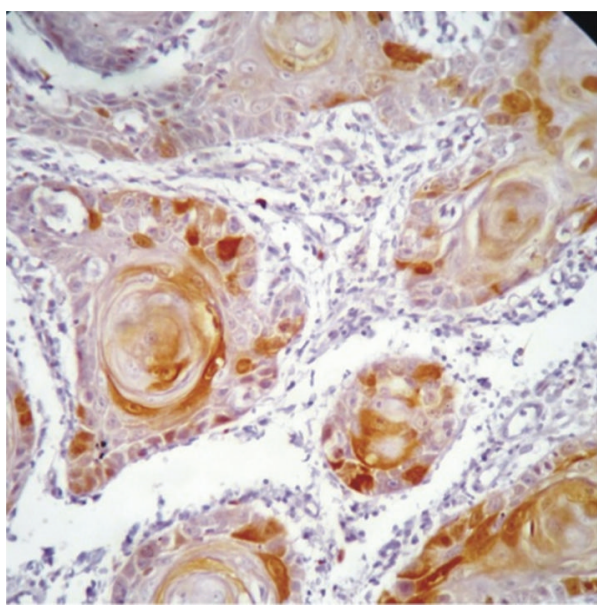

Positive control
HPV-16

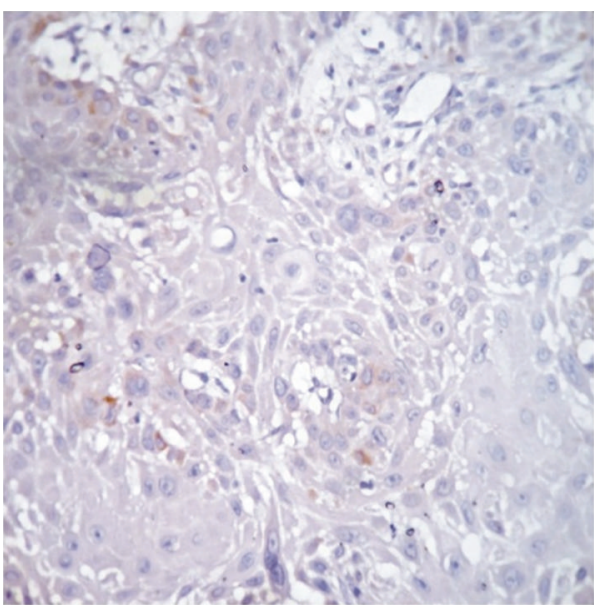

Negative control

(a)

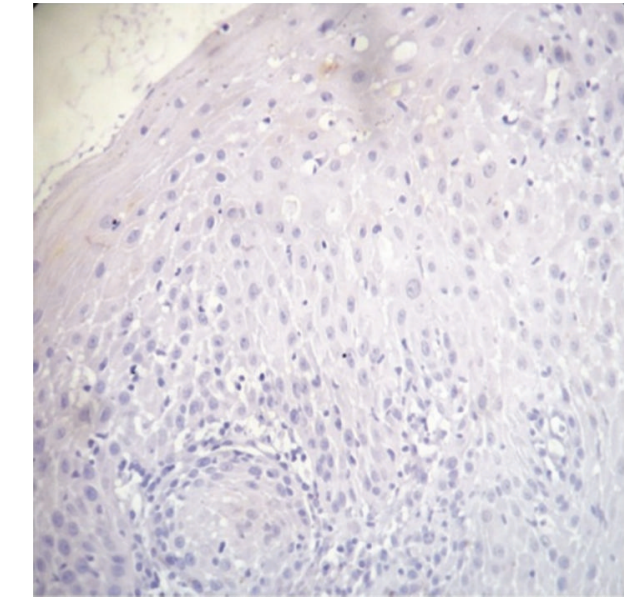

Negative control

(b)

FIgURE 1: Positive \& negative controls.

being positive for HPV 16. HPV 16 positivity was seen in buccal mucosa (50\%) \& alveolus (50\%) which was statistically significant.

HPV16 IHC showed a significant difference between proportions of mild \& severe grading of OED $(P<0.05)$, \& no significant difference was found between proportions of moderate grading for HPV 16 positivity.

All the patients were exposed to tobacco and mixed tobacco \& smoking habits. None of the patients were exposed to specific occupational or environmental carcinogens except tobacco (Table 1 and Figure 2).

4.2. HPV 16 Associations in Oral Squamous Cell Carcinoma. Study constituted 20 cases of OSCC (Table 1). The age range was $30 \mathrm{yrs}$ to $75 \mathrm{yrs}$ with mean age of 61.48 \& standard deviation of 7.21. Out of total cases, $35 \%$ cases fell in age group
TABLE 1: HPV positivity in oral epithelial dysplasia and OSCC.

\begin{tabular}{lcc}
\hline & HPV-16 positive (\%) & HPV 16 negative (\%) \\
\hline Epithelial dysplasia & $2(10 \%)$ & $18(90 \%)$ \\
OSCC & $7(35 \%)$ & $13(65 \%)$ \\
\hline Total & $9(22.5 \%)$ & $31(77.5 \%)$ \\
\hline
\end{tabular}

of 60 to 70 yrs. HPV 16 positivity was $57.14 \%$ in age group of 60 to $70 \mathrm{yrs}$. By applying chi-square test no significant association between age \& HPV 16 positive \& negative cases was found $(P>0.05)$.

The association between HPV 16 positivity, \& age was not statistically significant.

On applying $Z$ test of difference between two proportions there was a significant difference between proportions of 


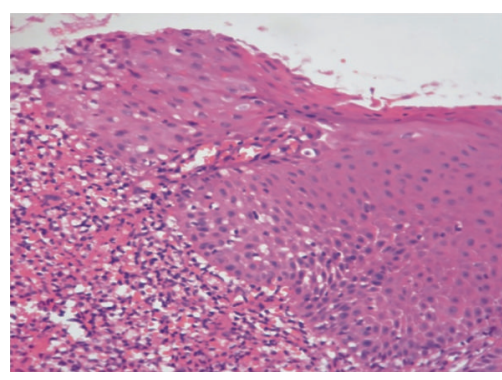

H \& E staining

(a)

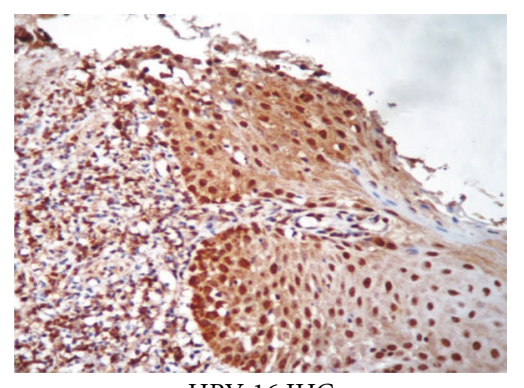

HPV-16 IHC

(b)

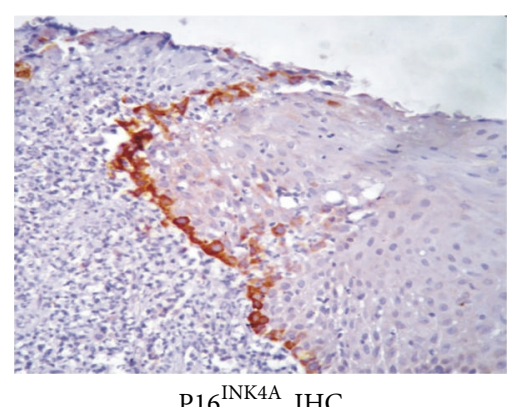

(c)

Figure 2: Oral epithelial dysplasia (400x).

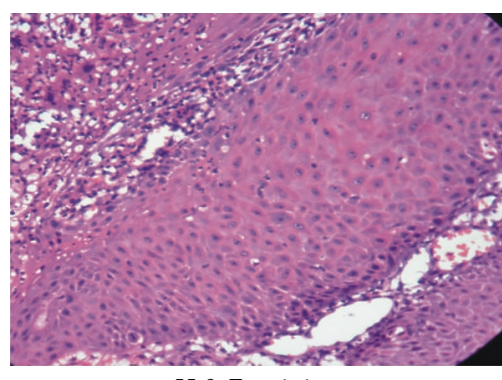

$\mathrm{H} \& \mathrm{E}$ staining

(a)

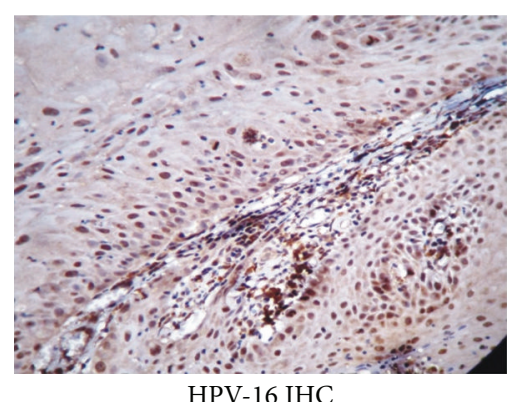

HPV-16 IHC

(b)

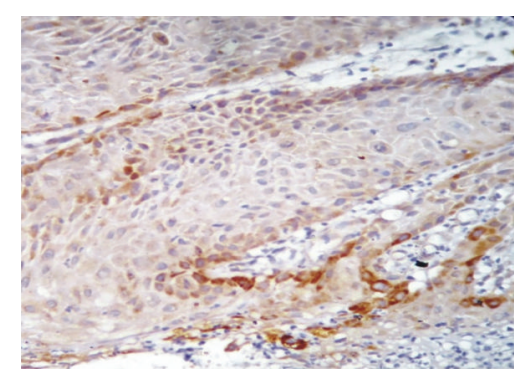

P16 ${ }^{\mathrm{INK} 4 \mathrm{~A}} \mathrm{IHC}$

(c)

FIgURE 3: Oral squamous cell carcinoma (400x).

TABLE 2: Histopathological grading of OSCC and HPV 16 positivity.

\begin{tabular}{lcc}
\hline $\begin{array}{l}\text { Degree of } \\
\text { differentiation }\end{array}$ & HPV 16 positive (\%) & HPV 16 negative (\%) \\
\hline Well & $5(71.44 \%)$ & $8(61.14 \%)$ \\
Moderate & $1(14.28 \%)$ & $4(30.77 \%)$ \\
Poor & $1(14.28 \%)$ & $1(7.69 \%)$ \\
\hline Total & $7(35 \%)$ & $13(65 \%)$ \\
\hline
\end{tabular}

$Z$ test, significant, $P<0.05$.

TABLE 3: TNM staging of oral squamous cell carcinoma.

\begin{tabular}{lc}
\hline Stage & Total number of cases (\%) \\
\hline Stage I & $9(45 \%)$ \\
Stage II & $7(35 \%)$ \\
Stage III & $4(20 \%)$ \\
Stage IV & 00 \\
\hline Total & 20 \\
\hline
\end{tabular}

lesion at lip, gingivobuccal sulcus \& retromolar triangle in HPV 16 positive and negative cases $(P<0.05)$. No significant difference was found between proportions of buccal mucosa \& alveolus in HPV16 positive \& HPV 16 negative cases $(P>$ $0.05)$.

It suggests that HPV 16 positivity in OSCC may be associated with sites like lip, gingivobuccal sulcus, and retromolar triangle.
Histopathological grading of OSCC cases showed degree of differentiation to be $13(65 \%)$ well, 5 (25\%) moderate, and 2 (10\%) poor. HPV 16 positivity on the basis of degree of differentiation was found to be $71.44 \%$ of well, $14.28 \%$ of moderate, and $14.28 \%$ of poor.

By applying $Z$ test of difference between two proportions statistically significant difference was found between proportions of histopathological grading $(P<0.05)$ (Table 2 and Figure 3).

Thus, HPV 16 positivity was found to be related to the degree of differentiation.

TNM staging of OSCC showed 9 cases (45\%) in Stage I, 7 cases (35\%) in Stage II, and 4 cases (20\%) in Stage III. HPV 16 association on TNM staging showed positivity in $51.15 \%$ of Stage I, $28.5 \%$ of Stage II, and $14.28 \%$ of Stage III. This finding was statistically significant after applying $Z$ test of difference (Table 3).

4.3. HPV 16 Positivity \& p16INK4A Expression. The study constituted total of 40 cases which included 20 cases of OED \& OSCC, respectively. All the 40 cases underwent IHC evaluation of HPV 16, out of which 9 cases were positive for HPV 16. 7 cases out of 9 HPV 16 positive cases, that is, $77.77 \%$, showed strong positivity for p16INK4A. German semiquantitative scoring system suggests that overall positivity for p16 was $60.62 \%$ which scores as 3 . It also suggests focal positivity for p16 by IHC. On the basis of the previous findings, immunohistochemical results are 
TABLE 4: P16 ${ }^{\text {INK4A }}$ immunohistochemistry in HPV 16 positive cases.

\begin{tabular}{|c|c|c|c|c|c|c|c|}
\hline \multirow{2}{*}{ Sr. no. } & \multirow{2}{*}{ Histopath. diagnosis } & \multicolumn{6}{|c|}{ P16 positivity in percentage (per $1 \mathrm{~cm}^{2}$ area) } \\
\hline & & Area 1 & Area 2 & Area 3 & Area 4 & Area 5 & Avg. (\%) \\
\hline (1) & OED & 54.90 & 50.0 & 71.95 & 60.0 & 65.0 & 60.37 \\
\hline (2) & OED & 5.2 & 6.7 & 7.8 & 9.3 & 2.4 & 6.28 \\
\hline (3) & WDOSCC & 56.99 & 62.21 & 59.98 & 63.04 & 60.88 & 60.62 \\
\hline (4) & WDOSCC & 0.0 & 0.0 & 1.3 & 0.0 & 2.5 & 0.76 \\
\hline (5) & WDOSCC & 68.36 & 46.83 & 80.0 & 76.92 & 61.22 & 66.66 \\
\hline (6) & WDOSCC & 63.56 & 64.21 & 63.33 & 58.39 & 59.57 & 60.81 \\
\hline (7) & WDOSCC & 0.0 & 0.0 & 0.0 & 0.0 & 0.0 & 0.0 \\
\hline (8) & MDOSCC & 60.77 & 65.79 & 64.44 & 57.05 & 59.74 & 61.56 \\
\hline (9) & PDOSCC & 14.47 & 24.35 & 32.5 & 51.17 & 27.43 & 29.98 \\
\hline
\end{tabular}

Overall mean and SD of all 9 slides are $60.62 \%$ and $12.56 \%$, respectively.

suggestive of strong correlation between positivity of HPV 16 and pl6INK4A (Table 4, Figures 2 and 3).

\section{Discussion}

The mechanism which leads to development of malignancy is always difficult to evaluate. Transformation of normal tissue into malignancy occurs through intermediate stages of premalignancy. The etiology or risk factors for initiation development of malignancy and always been a challenge for investigators. Tobacco and alcohol consumption is the most common risk factor for developing oropharyngeal cancers. In addition to this, certain viral infections play an important role in malignancy $[4,8,21]$. The participation of HPV in oral and oropharyngeal carcinoma was first proposed by K. Syrjänen et al. [22] in 1983 and then supported by several other authors $[8,9,23,24]$. According to the studies carried out to explain etiological role of HPV in cancer of the head and neck region, HPV has been found to be both in an episomal form and in an integral form. It has also been suggested that HPV may be latent for a long time in the episomal format in the oral mucosa, hence being responsible for initiation and development of a tumoral growth. This tumoral growth may occur as a result of a multicarcinogenic interaction together with some other carcinogens and cocarcinogens $[3,5,12]$. K. Syrjänen et al. in 1983 first demonstrated HPV 16 DNA in 6 of 21 cases of OED [22]. Later, one more study by them in 1986 found HPV 16 antigen expression in $66.7 \%$ of cases of leukoplakia with majority of cases with mild dysplasia [11].

Another study by Fregonesi et al. found HPV 16/18 positivity of $40 \%$ in oral premalignant lesions with various grades of dysplasia [24]. Shroyer investigated cases of OED for presence of HPV $16 / 18$ as one of the risk factors for carcinogenesis. Their results showed positivity of 17\% [25]. Some investigators like Rafik Abdelsayed [26] (0\%) and Carmen Maria Lazzari et al. (8.8\%) found no significant association between epithelial dysplasia and HPV status. Recently, systematic review from 1966 to 2010 by S. Syrjänen et al., expressed statisfactory significant association between HPV 16 positivity and OED ranging from $18.8 \%$ to $48.4 \%$ [27]. There was high variability of HPV positivity in OED ranging between $0 \%$ to $80 \%$ by various investigators [11, 22 , $27,28]$.

Our study showed $2 / 20$, that is, $10 \%$ HPV 16 positivity in OED, which is very low but in accordance with the literature.

There are more than $40 \mathrm{HPV}$ types, which were detected in anogenital invasive tumors and their precursor lesions. The occurrence of low risk type HPV in precursor lesions rarely progresses to malignancy. Therefore, HR HPV like types 16 and 18 has major contribution in papillomavirus-associated carcinogenesis $[12,19,20]$.

In 1983 K. Syrjänen et al. [22] detected HPV positivity in $46 \%[14 / 30]$ of cases of oral and oropharyngeal SCC by using IHC. D'Souza et al. [29] also showed presence of HPV 16 association with oropharyngeal carcinoma with positivity of $64 \%$. Li et al. [30] studied association between tonsil carcinoma and HPV, showing $42 \%$ positivity, whereas Schlecht et al. [31] found it to be $29 \%$ including HNSCC. The majority of the literature on association between HPV \& OSCC is based on wide array of molecular assays like IHC, ISH, \& PCR. All the data pertaining to this finding showed positivity for HPV in wide range of variability from $0 \%$ to $100 \%[5,7,8,19,20,22,27,28,32-35]$. Kenneth Shroyer et al. [25] (10\%), Fregonesi et al. [24] (11\%), Cunnigham et al. [17] (14.6\%), and Syrjänen et al. [23] (50\%) studied carcinogenesis mediated through HPV 16 and concluded that HPV 16 has possible etiologic role in development of OSCC.

In contradiction to the previous studies, Young and Min et al. [32] and Tsuchiya et al. [36] were unable to find such association between HPV 16 and OSCC. Recently, systematic review by Syrjänen et al. [27] in 2011 in their review from 1966 to 2010 showed strong correlation between the presence of HPV DNA, specifically with HPV16 and OSCC. Estimated risk for HPV was found to vary from 0.32 to 363.0 in 33 studies included by them. In our study $35 \%$ (7/20) of OSCC cases were positive for HPV 16 by IHC, which is in accordance with the literature review so far. In current study, agewise distribution of HPV 16 positive and negative cases was not showing any statistically significant correlation which is in accordance with Kurose et al. [37] and in contradiction to Cruz et al. [38] In genderwise distribution of HPV positive OSCC cases, Werness et al. [13] found statistical correlation with male predominance. Other investigators like Cruz et 
al. [38] and Priya Koppikar et al. [5] concluded that men were more likely to be positive for HPV than females. Our study had no such correlation related to gender. Sitewise distribution of OSCC cases amongst HPV16 positive cases in our study showed no significant correlation, which is in accordance with Jalal et al. [39] \& Cruz et al. [38] In the current study we found that there was significant association between HPV 16 and histopathologic grades of differentiation, with HPV 16 positivity being highest in cases of WDSCC (71.44\%). Our results are in accordance with Abdelsayed [26] and in contradiction to Schlecht et al. [31] and Badaracco et al. [34] HPV 16 association in OSCC with clinical staging was studied by several investigators like Mellin et al. [20], Tachezy et al. [7], Badaracco et al. [34], and so forth. Out of them Mellin et al. [20] found significant correlation between TNM staging and HPV positivity. Our results are in accordance with the literature review, showing majority of positive cases from Stage I (51.44\%).

Establishment of exact HPV association in carcinogenesis has always been a challenge due to absence of universal standardization detection method. The variety of methods in current use include PCR, ISH, detection of serum antibodies directed against HPV epitope, IHC for detection of HPV ORF like $\mathrm{L}_{1}, \mathrm{E}_{6}, \mathrm{E}_{7}$, and p16 immunohistochemistry. Any single method of detection has some limitations. Thus use of algorithms that combine the strength of complementary assay is needed. According to Guimaraes et al. [40], Fregonesi et al. [24], Cunningham et al. [17], Negri et al. [28], and Samama et al. [40] overexpression of p16INK4A protein by immunohistochemistry can act as surrogate biomarker of $\mathrm{HPV}$-induced carcinomas.

In accordance with the review so far, our study showed a strong correlation between HPV positivity and disruption of regulation of p16INK4A suppressor protein which resulted in overexpression of p16INK4A in OED (50\%) and OSCC (85.71\%). We also found that overall $60.62 \%$ cells showed positive expression of p16INK4A in those cases. This suggests focal positivity for p16INK4A which has an association with carcinogenesis by HR HPV [24, 40].

This clearly reveals that pl6INK4A immunohistochemistry can be used as surrogate biomarker for HPV-associated carcinogenesis.

\section{Conclusion}

Immunohistochemical detection of HPV 16 and p16INK4A was performed using Super Sensitive Polymer HRP IHC detection system (Biogenix, CA). Overall 22.5\% of cases in our study were found to be positive for HPV 16 which includes $10 \%$ of cases of OED and $35 \%$ of cases of OSCC. A significant correlation was found in degree of differentiation of tumor and TNM staging amongst HPV 16 positive and negative cases. Amongst the HPV 16 positive cases, more than $60 \%$ of cells were positive for p16INK4A IHC in OED (50\%) and OSCC $(85.71 \%)$. There are a wide range of molecular assays available for the detection of HPV which includes IHC, ISH, PCR, Southern blotting, and p16INK4A IHC. All these methods have some advantages as well as disadvantages.
Immunohistochemical detection of HPV 16 is based on viral integration in tissue. It mainly detects viral capsid proteins of papillomavirus.

HPV IHC lacks sensitivity. So an attempt was made in our study to combine specificity of HPV 16 IHC with sensitivity of p16INK4A IHC for detection of HPV infection in OED \& OSCC.

On the basis of the previous relation we can conclude that,

(i) HPV 16 does have a role in pathogenesis of OED and OSCC and can be studied effectively by immunohistochemistry;

(ii) HPV 16 participates in disruption of the regulation of p16INK4A suppressor protein and thus can used as surrogate biomarker for detection of HPV infection in OED \& OSCC.

\section{References}

[1] L. Koss, Koss's Diagnostic Cytology, vol. 1, Lippincott Williams \& Wilkins, Philadelphia, Pa, USA, 5th edition, 2006.

[2] D. M. Parkin, F. Bray, J. Ferlay, and P. Pisani, "Global cancer statistics, 2002," CA Cancer Journal for Clinicians, vol. 55, no. 2, pp. 74-108, 2005.

[3] D. Adelstein, J. A. Ridge, M. L. Gillison et al., "Head and neck squamous cell carcinoma \& HPV: Summary of national cancer institute state of the science meeting," Head \& Neck, vol. 31, no. 11, pp. 1393-1422, 2009.

[4] R. Rajendran and B. Shivapathasundharam, Eds., Shafers Textbook of Oral Pathology, Elseviers, New York, NY, USA, 6th edition, 2009.

[5] P. Koppikar, E. M. De Villiers, and R. Mulherkar, "Identification of human papillomaviruses in tumors of the oral cavity in an Indian community," International Journal of Cancer, vol. 113, no. 6, pp. 946-950, 2005.

[6] S. Nair and M. R. Pillai, "Human papillomavirus and disease mechanisms: relevance to oral and cervical cancers," Oral Diseases, vol. 11, no. 6, pp. 350-359, 2005.

[7] R. Tachezy, J. Klozar, M. Saláková et al., "HPV and other risk factors of oral cavity/oropharyngeal cancer in the Czech Republic," Oral Diseases, vol. 11, no. 3, pp. 181-185, 2005.

[8] C. Scully, M. F. Cox, S. S. Prime, and N. J. Maitland, "Papillomaviruses: the current status in relation to oral disease," Oral Surgery Oral Medicine and Oral Pathology, vol. 65, no. 5, pp. 526-532, 1988.

[9] R. O. Greer, K. L. Schroeder, and L. Crosby, "Morphologic and immunohistochemical evidence of human papillomavirus capsid antigen in smokeless tobacco keratoses from juveniles and adults," Journal of Oral and Maxillofacial Surgery, vol. 46, no. 11, pp. 919-929, 1988.

[10] P. K. Ha and J. A. Califano, "The role of human papillomavirus in oral carcinogenesis," Critical Reviews in Oral Biology and Medicine, vol. 15, no. 4, pp. 188-196, 2004.

[11] M. Hoffmann, C. Lohrey, A. Hunziker, T. Kahn, and E. Schwarz, "Human papillomavirus type 16 E6 and E7 genotypes in headand-neck carcinomas," Oral Oncology, vol. 40, no. 5, pp. 520524, 2004.

[12] K. Milde-Langosch, S. Riethdorf, A. Kraus-Pöppinghaus, L. Riethdorf, and T. Löning, "Expression of cyclin-dependent 
kinase inhibitors p16MTS1, p21WAF1, and p27KIP1 in HPVpositive and HPV-negative cervical adenocarcinomas," Virchows Archiv, vol. 439, no. 1, pp. 55-61, 2001.

[13] B. A. Werness, A. J. Levine, and P. M. Howley, "Association of human papillomavirus types 16 and 18 E6 proteins with p53," Science, vol. 248, no. 4951, pp. 76-79, 1990.

[14] R. Ralham, "Diagnostic potential of genomic and proteomic signatures in oral cancer," International Journal of Human Genetics, vol. 7, no. 1, pp. 57-66, 2007.

[15] A. K. Chaudhary, M. Singh, S. Sundaram, and R. Mehrotra, "Role of human papillomavirus and its detection in potentially malignant and malignant head and neck lesions: updated review," Head \& Neck Oncology, vol. 1, p. 22, 2009.

[16] C. L. Koo, L. F. Kok, M. Y. Lee et al., "Scoring mechanisms of p $16^{\text {INK4a }}$ immunohistochemistry based on either independent nucleic stain or mixed cytoplasmic with nucleic expression can significantly signal to distinguish between endocervical and endometrial adenocarcinomas in a tissue microarray study," Journal of Translational Medicine, vol. 7, p. 25, 2009.

[17] L. L. Cunningham, G. M. Pagano, M. Li et al., "Overexpression of $\mathrm{p} 16^{\mathrm{INK} 4 \mathrm{a}}$ is a reliable marker of human papillomavirusinduced oral high-grade squamous dysplasia," Oral Surgery, Oral Medicine, Oral Pathology, Oral Radiology and Endodontology, vol. 102, no. 1, pp. 77-81, 2006.

[18] S. Warnakulasuriya, N. W. Johnson, and I. Van Der Waal, "Nomenclature and classification of potentially malignant disorders of the oral mucosa," Journal of Oral Pathology and Medicine, vol. 36, no. 10, pp. 575-580, 2007.

[19] L. Barnes, J. W. Eveson, P. Reichart, and D. Sidransky, Eds., WHO Classification of Tumours: Pathology and Genetics of Head and Neck Tumours, ARC Press, Lyon, France, 2005.

[20] H. Mellin, S. Friesland, R. Lewensohn, T. Dalianis, and E. Munck-Wikland, "Human papillomavirus (HPV) DNA in tonsillar cancer: clinical correlates, risk of relapse, and survival," International Journal of Cancer, vol. 89, no. 3, pp. 300-304, 2000.

[21] P. Shah, Text Book of Oral Cancer, Martin Duntitz, London, UK, 2003.

[22] K. Syrjänen, S. Syrjanen, and M. Lamberg, "Morphological and immunohistochemical evidence suggesting human papillomavirus (HPV) involvement in oral squamous cell carcinogenesis," International Journal of Oral Surgery, vol. 12, no. 6, pp. 418-424, 1983.

[23] S. M. Syrjänen, K. J. Syrjanen, and M. A. Lamberg, "Detection of human papillomavirus DNA in oral mucosal lesions using in situ DNA-hybridization applied on paraffin sections," Oral Surgery Oral Medicine and Oral Pathology, vol. 62, no. 6, pp. 660-667, 1986.

[24] P. A. G. Fregonesi, D. B. Teresa, R. A. Duarte, C. B. Neto, M. R. B. De Oliveira, and C. P. Soares, "p16 ${ }^{\text {INK4a }}$ immunohistochemical overexpression in premalignant and malignant oral lesions infected with human papillomavirus," Journal of Histochemistry and Cytochemistry, vol. 51, no. 10, pp. 1291-1297, 2003.

[25] K. R. Shroyer and R. O. Greer, "Detection of human papillomavirus DNA by in situ DNA hybridization and polymerase chain reaction in premalignant and malignant oral lesions," Oral Surgery Oral Medicine and Oral Pathology, vol. 71, no. 6, pp. 708-13, 1991.

[26] R. A. Abdelsayed, "Study of human papillomavirus in oral epithelial dysplasia and epidermoid carcinoma in the absence of tobacco and alcohol use," Oral Surgery, Oral Medicine, Oral Pathology, vol. 71, no. 6, pp. 730-732, 1991.
[27] S. Syrjänen, G. Lodi, I. von Bültzingslöwen et al., "Human papillomaviruses in oral carcinoma and oral potentially malignant disorders: a systematic review," Oral Diseases, vol. 17, no. 1, pp. 58-72, 2011.

[28] G. Negri, G. Bellisano, G. F. Zannoni et al., "p16 ${ }^{\text {INK4a }}$ and HPV L1 immunohistochemistry is helpful for estimating the behavior of low-grade dysplastic lesions of the cervix uteri," American Journal of Surgical Pathology, vol. 32, no. 11, pp. 1715-1720, 2008.

[29] G. D’Souza, A. R. Kreimer, R. Viscidi et al., "Case-control study of human papillomavirus and oropharyngeal cancer," The New England Journal of Medicine, vol. 356, pp. 1944-1956, 2007.

[30] W. Li, C. H. Thompson, Y. E. Cossart et al., "The expression of key cell cycle markers and presence of human papillomavirus in squamous cell carcinoma of the tonsil," Head and Neck, vol. 26, no. 1, pp. 1-9, 2004.

[31] N. F. Schlecht, R. D. Burk, L. Adrien et al., "Gene expression profiles in HPV-infected head and neck cancer," Journal of Pathology, vol. 213, no. 3, pp. 283-293, 2007.

[32] S. K. Young and K. W. Min, "In situ DNA hybridization analysis of oral papillomas, leukoplakias, and carcinomas for human papillomavirus," Oral Surgery Oral Medicine and Oral Pathology, vol. 71, no. 6, pp. 726-9, 1991.

[33] R. G. McKaig, R. S. Baric, and A. F. Olshan, "Human papillomavirus and head and neck cancer: epidemiology and molecular biology," Head Neck, vol. 20, no. 3, pp. 250-265, 1998.

[34] G. Badaracco, A. Venuti, A. Bartolazzi, R. Morello, F. Marzetti, and M. L. Marcante, "Overexpression of p53 and bcl-2 proteins and the presence of HPV infection are independent events in head and neck cancer," Journal of Oral Pathology and Medicine, vol. 29, no. 4, pp. 173-179, 2000.

[35] W. Li, C. H. Thompson, Y. E. Cossart et al., "The expression of key cell cycle markers and presence of human papillomavirus in squamous cell carcinoma of the tonsil," Head and Neck, vol. 26, no. 1, pp. 1-9, 2004.

[36] H. Tsuchiya, Y. Tomita, H. Shirasawa, H. Tanzawa, K. Sato, and B. Simizu, "Detection of human papillomavirus in head and neck tumors with DNA hybridization and immunohistochemical analysis," Oral Surgery Oral Medicine and Oral Pathology, vol. 71, no. 6, pp. 721-5, 1991.

[37] K. Kurose, M. Terai, N. Soedarsono et al., "Low prevalence of HPV infection and its natural history in normal oral mucosa among volunteers on Miyako Island, Japan," Oral Surgery, Oral Medicine, Oral Pathology, Oral Radiology and Endodontology, vol. 98, no. 1, pp. 91-96, 2004.

[38] I. B. F. Cruz, P. J. F. Snijders, R. D. M. Steenbergen et al., "Agedependence of human papillomavirus DNA presence in oral squamous cell carcinomas," European Journal of Cancer B, vol. 32, no. 1, pp. 55-62, 1996.

[39] H. Jalal, C. M. Sanders, S. S. Prime, C. Scully, and N. J. Maitland, "Detection of human pappiloma virus type 16 DNA in oral squames from normal young adults," Journal of Oral Pathology and Medicine, vol. 21, no. 10, pp. 465-470, 1992.

[40] B. Samama, D. Lipsker, and N. Boehm, "p16 expression in relation to human papillomavirus in anogenital lesions," Human Pathology, vol. 37, no. 5, pp. 513-519, 2006. 


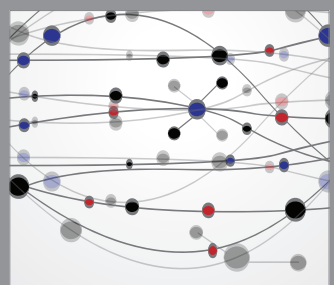

The Scientific World Journal
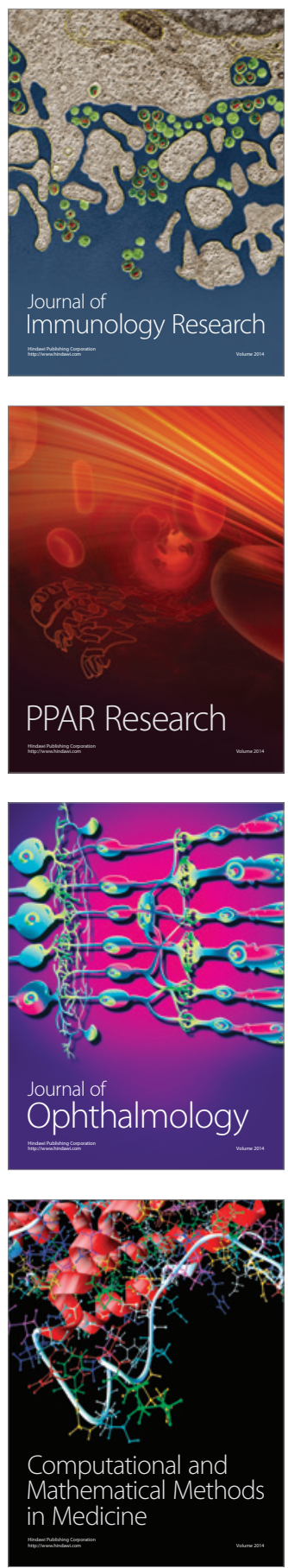

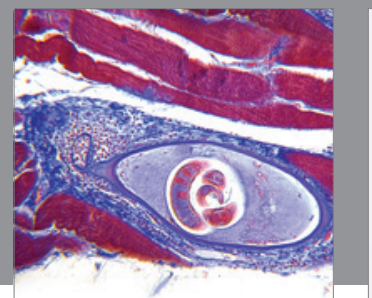

Gastroenterology

Research and Practice
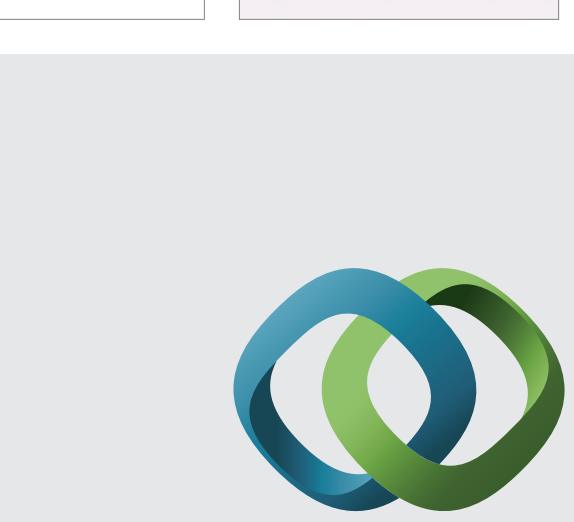

\section{Hindawi}

Submit your manuscripts at

http://www.hindawi.com
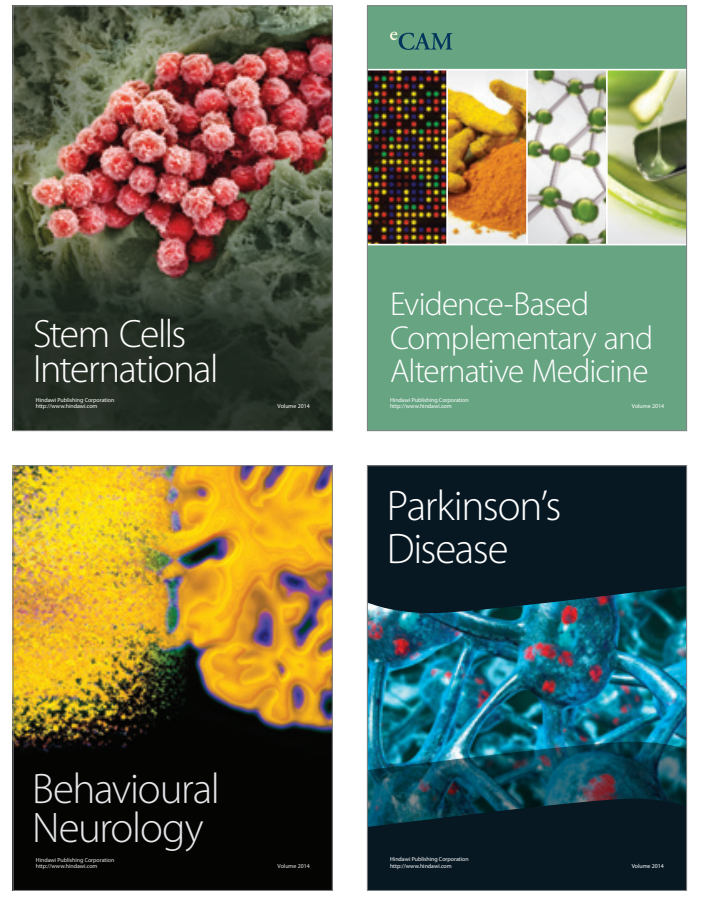
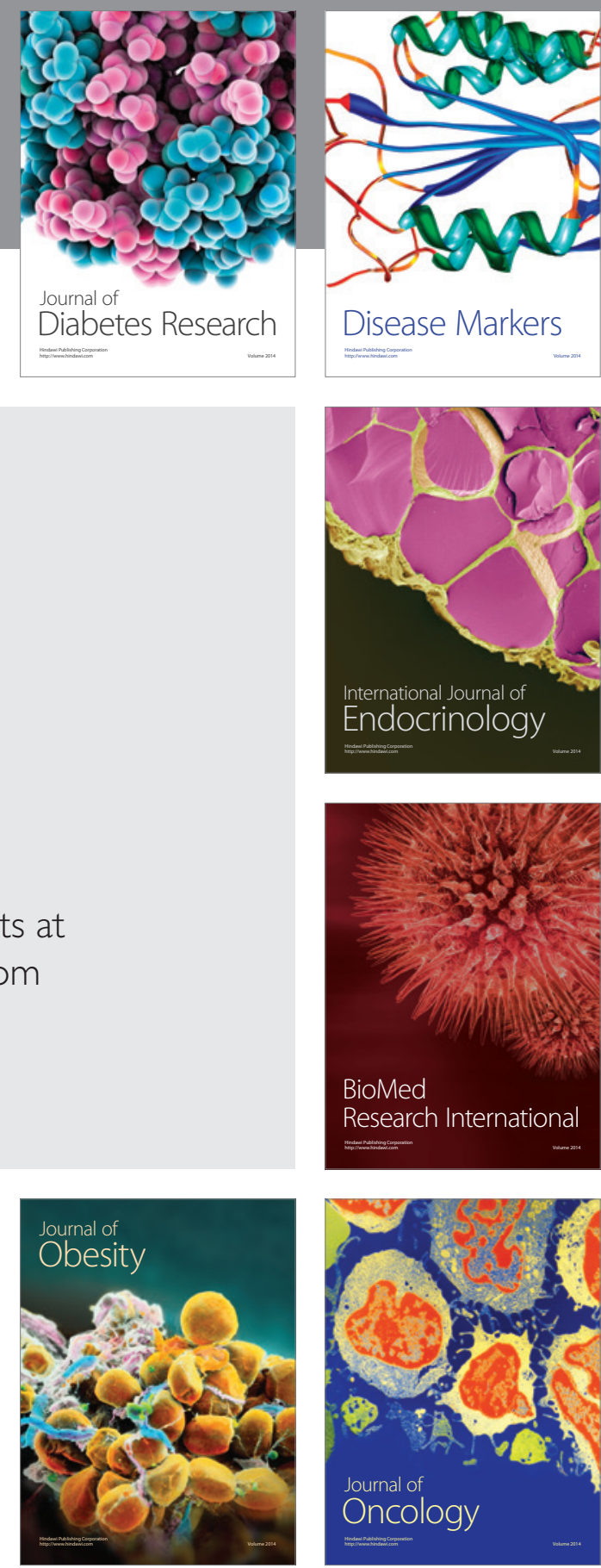

Disease Markers
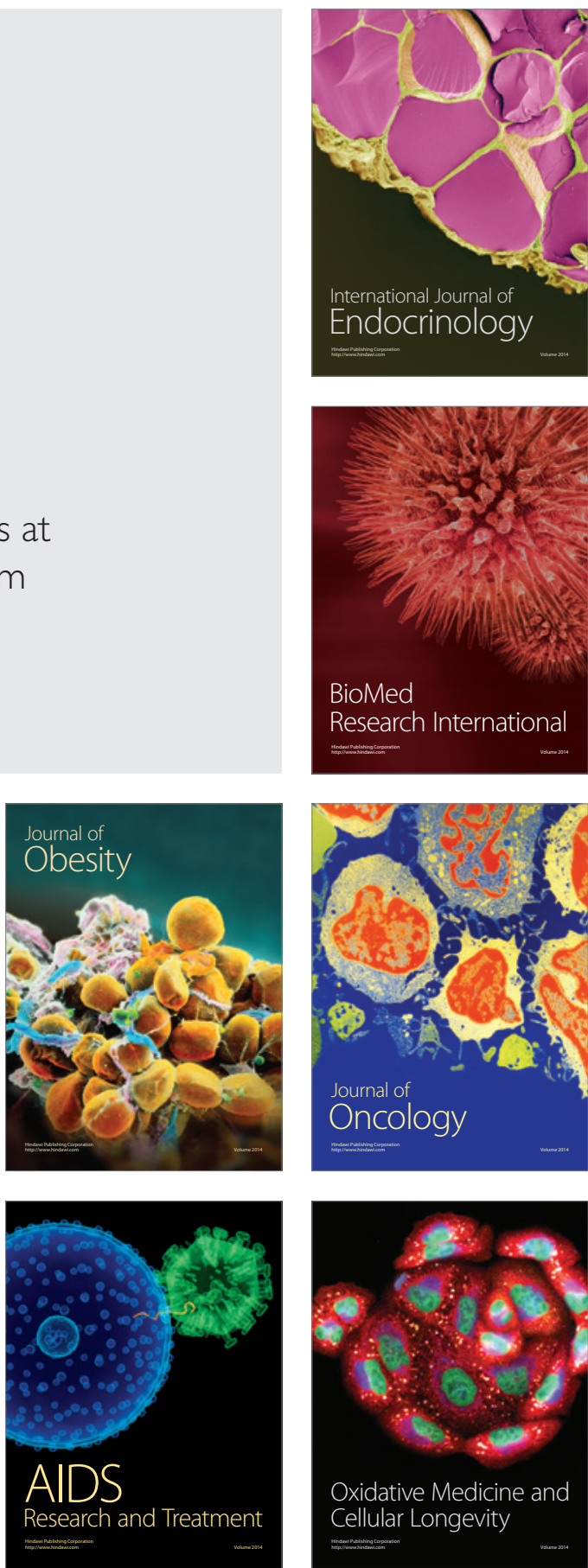